\title{
Pattern and Predicting Risk Factors of Multi-Morbidity in the AzarCohort Population Using Structural Equation Model
}

\author{
Mohammad-Hossein Somi ${ }^{1}$, Alireza Ostadrahimi², Neda Gilani ${ }^{3}$, Arash Haji Kamanaj ${ }^{2}$, \\ Sina Hassannezhad ${ }^{2}$, and Elnaz Faramarzi ${ }^{2}$ \\ ${ }^{1}$ Tabriz University of Medical Sciences \\ ${ }^{2}$ Affiliation not available \\ ${ }^{3}$ Department of Statistics and Epidemiology, Faculty of Health, Tabriz University of \\ Medical Sciences, Tabriz, Iran.
}

June 6, 2021

\begin{abstract}
Background: The co-existence of chronic diseases (CD), a condition defined as multimorbidity (MM), is becoming a major public health issue. Understanding the general framework of MM diseases according to the well-known risk factors can assist in finding direct and indirect relationships among them. Therefore, we aimed to determine pattern and predicting risk factors of multimorbidity in the Azar Cohort population using Structural Equation Model (SEM). Methods: In this study, the prevalence of MM in 15006 XXX cohort population was evaluated. MM was defined as the co-existence of two or more CDs. The information regarding socio-economic, demographic, sleeping habits, and physical activity were collected by questionnaires. A multi-group SEM was employed to model complex relationships between directly- and indirectly-observed variables. Results: The overall MM was seen in $28.8 \%$ of the population. The most prevalent chronic diseases were obesity, hypertension, depression, and diabetes, respectively. Obesity, depression, and diabetes were the most co-occurring CDs in our population. The SEM diagram indicated the overall effect of socio-demographic (predictors) and sleep and physical activity (mediators) on the number of CDs. The number of CDs in the active participants and those who sleep 6.6-7.3 hours/day was lower than the inactive participants and those who sleep [?]6.5 hours/day. Conclusions: According to our results, it seems that the reduction of MM is possible through promoting public health from an early age and for a wide range of socio-economic conditions, provided that the necessary support for general health is offered for the aging population.
\end{abstract}

Pattern and Predicting Risk Factors of Multi-Morbidity in the XXX Cohort Population Using Structural Equation Model

Abstract

Aims: The aims of this study were to determine pattern and predicting risk factors of multi-morbidity in the Azar Cohort population using Structural Equation Model (SEM).

Methods: In this study, the prevalence of MM in 15006 XXX cohort population was evaluated. MM was defined as the co-existence of two or more CDs. The information regarding socio-economic, demographic, sleeping habits, and physical activity were collected by questionnaires. A multi-group SEM was employed to model complex relationships between directly- and indirectly-observed variables.

Results: The overall MM was seen in $28.8 \%$ of the population. The most prevalent chronic diseases were obesity, hypertension, depression, and diabetes, respectively. Obesity, depression, and diabetes were the most co-occurring CDs in our population. The SEM diagram indicated the overall effect of socio-demographic (predictors) and sleep and physical activity (mediators) on the number of CDs. The number of CDs in the 
active participants and those who sleep 6.6-7.3 hours/day was lower than the inactive participants and those who sleep [?] 6.5 hours/day.

Conclusions: According to our results, it seems that the reduction of MM is possible through promoting public health from an early age and for a wide range of socio-economic conditions, provided that the necessary support for general health is offered for the aging population.

Keywords: Chronic disease, Mmultimorbidity, Structural Equation Model, cohort study

What's known?

-Many of the risk factors (including age, obesity, smoking, and hypertension) are linked with multi morbidity $(\mathrm{MM})$.

- Cohort studies show a direct association between high age and low socioeconomic populations, with a growing trend in women.

- A large number of cohort MM studies have focused on old ages, especially those over 65.

- Studies that evaluated the predictor risk factors of MM using the Structural Equation Model (SEM) are rare.

What's new?

-In our study, using SEM method, physical activity and sleeping habits are mediators, while wealth score index, the number of years of schooling, marital status, and residence regions (rural) influence the number of chronic diseases through physical activity and sleeping habits.

- Another finding in this study indicated depression as the pivotal factor in multidisciplinary patterns, which is closely associated with an increased risk of chronic diseases.

Introduction:

Multimorbidity (MM) is often described as the co-existence of two or more chronic diseases, including physical non-communicable long-term conditions and mental health problems ${ }^{1}$. The prevalence of MM is reported to vary from $12.9 \%$ in the general population to $95.1 \%$ in older people. Many of the risk factors (including age, obesity, smoking, and hypertension) are linked with multimorbidity ${ }^{2}$. In other words, each factor can result in co-morbidities, yield vulnerable conditions for patients, and establish a generally unfavorable quality of life $^{3}$. Over the past decades, with improving health-care systems and increased life expectancy, the average age has gradually increased. However, as people move toward adulthood, MM and related complications are more likely to affect individuals. Consequently, due to enduring diseases for long periods of time and increasing age, people experience mental, emotional, and physical weakness. This simultaneous endurance of physical and mental hardships causes excessive fatigue that prevents people from having physical activity, which in turn, influences social participation and cognitive health ${ }^{4}$. Therefore, this chain of risk factors has increased the prevalence rate of non-communicable diseases in recent decades, despite the advancement of diagnostic and screening capabilities.

The most common classification method to study the prevalence of MM is based on demographic characteristics such as age, sex, level of income, and socioeconomic status ${ }^{5}$. Cohort studies show a direct association between high age and low socioeconomic populations, with a growing trend in women ${ }^{3}$. Because of continuous monitoring requirements, MM patients are imposed to overwhelming treatments, frequent hospitalizations, and polypharmacy ${ }^{6}$. In specific, polypharmacy is a significant risk factor that results in a vicious cycle and more risk factors by increasing hospitalizations and presentation of new multimorbidities, such as liver disease due to the disruption of liver enzymes ${ }^{6,7}$.

Several studies investigated the relationship between physical activity and the emergence of MM in different populations. A study showed that the requirement of polypharmacy in MM patients with low levels of physical activity is doubled in comparison with active patients. Moreover, decreased physical exercise negatively 
impacts the lipid profile and insulin sensitivity and could develop anxiety and depression in COPDs ${ }^{8}$. In one of these studies, the relationship between watching television and physical activity was examined. As demonstrated in this study, increasing the hours of watching television is directly associated with a rising prevalence of multimorbidity. In other words, decreasing physical activity enhances the influence of sedentary behaviors ${ }^{9}$. Another study on the Chinese adult population by Wang et al. demonstrated that inadequate sleeping alters the natural metabolism of the body, which plays a key role in the emergence of MMs such as cerebrovascular disease, cardiovascular disease, and diabetes. However, this study found no association between sleep duration, anemia, and chronic kidney disease ${ }^{10}$.

Understanding the general framework of MM in accordance with well-known risk factors can assist in finding direct and indirect relationships between them. This will enable predicting their occurrence sequence in people with different features. Moreover, using this information, we will be able to identify and prevent the defective cycles of the diseases at an appropriate and reversible point ${ }^{11}$.

However, these complex demands increase the workload of health-care centers and create unnecessary expenses for the system and patients. Thus, managing the prevalence of MM in patients is among the most crucial challenges in modern medicine and is a global research priority ${ }^{12}$.

Nevertheless, despite its critical significance, there are many profound gaps in the exact relationships between each demographic factor and multimorbidity. Usually, age and sex are considered as demographic characteristics to express the prevalence of multimorbidity ${ }^{11}$.

A large number of cohort MM studies have focused on old ages, especially those over 65 . Therefore, it is impossible to determine the behavioral pattern of MMs in primary ages. In addition, these studies have investigated a few specific diseases, and no investigation had been carried out to clarify their relationship with other diseases ${ }^{13}$.

Therefore, the purpose of this study is to determine the prevalence of MM by considering all demographic factors to express a comprehensive relationship pattern between various diseases (such as cardiovascular diseases, cancers, asthma, and other frequent diseases) and their risk factors, using a structural equation modeling approach in the XXX cohort population.

Method and Material:

In the following cross-sectional study, the prevalence of MM in 15006 subjects who participated in the XXX cohort study was evaluated. XXX cohort study is part of a large prospective epidemiological research studies in XXX (XXX cohort) ${ }^{14}$ and has been approved by the Ethical Committee of XXX University of medical sciences (XXXmed.Rec.1393.205). The pilot and the enrollment phases for this study was launched in 2014, and it was concluded in 2017. Comprehensive details about the XXXcohort study are provided in other published article ${ }^{15}$.

MM is defined as the co-existence of two or more Chronic Diseases (CD), including hypertension, diabetes, cardiovascular diseases (CVD), cerebrovascular diseases, asthma, cancers (gastrointestinal, breast, prostate, skin, bladder, lung, head and neck, and hematopoietic), depression, fatty livers, rheumatoid disease, and obesity.

In the questionnaires, participants were considered to have these diseases when they answered yes to the following question: "Has any doctor ever told you that you have -?" Moreover, obesity was defined as a body mass index of $30 \mathrm{~kg} / \mathrm{m}^{2}$ or higher.

Anthropometric Measurements:

The weight and height of all subjects were measured, and the body mass index was determined using the standard formula: weight $(\mathrm{kg}) /$ height $\left(\mathrm{m}^{2}\right)$. The anthropometric measurements are described in detail elsewhere ${ }^{14}$. Information regarding age, gender, education level, marital status, smoking status, and sleep habits were collected using the questionnaires. 
Socioeconomic status was evaluated using Wealth Score Index (WSI), which is calculated by Multiple Correspondence Analysis (MCA). Ownership of a variety of durable assets (e.g., dishwasher, car, and television), household condition (e.g., the number of rooms, type of ownership), and education level were used in the calculation of WSI for each participant. Participants of the study were categorized into five SES quintiles, from the lowest (1st quintile) to the highest (5th quintile). In this study, the daily activity of the participants was evaluated using a questionnaire recorded by the participants. For this purpose, a criterion called MET has been employed. Each MET is equal to the amount of energy that each person consumes relative to their weight. For instance, one MET is equal to the amount of oxygen used by each person while resting per kilogram of their body weight per minute, which is $3.5 \mathrm{ml}$ of oxygen, and 4 MET equals 16 milliliters of oxygen used per kilogram of their body weight per minute. Through this criterion, we obtained the level of activity based on its respective MET for each person.

Statistical Analysis:

In this study, the STATA software (version 16, Stata Corp, College Station, Texas) was employed for data analysis. The normality of data was assessed using the Kolmogorov-Smirnov test and descriptive statistics. The mean (standard deviation) was reported for the quantitative data, and the frequency (percentage) was reported for qualitative data. In the current analysis, quantitative variables were age, BMI, while qualitative variables were gender, marital status, education level, residential region, smoking status, WSI, and sleep duration. One-Way ANOVA, chi-square, and Kruskal-Wallis H were used to compare quantitative, qualitative, and categorical qualitative variables, respectively, between multimorbidity classifications. The MMs were classified into four groups: 0 (no chronic diseases), 1 (one chronic disease), 2 (two chronic diseases), 3 (3 chronic diseases), and 4 ([?] four chronic diseases). Moreover, WSI, METS, and sleep duration were categorized into tertile, quartile, and quintiles, respectively.

A multi-group Structural Equation Model (SEM) was employed to model complex relationships between directly- and indirectly-observed variables. In this model, MM was considered as multiple chronic diseases. Age, gender, marital status, WSI, the number of years in school, residence regions, and current smoking status were predictors, sleeping habits and physical activity were the moderators, and the number of chronic diseases was the outcome. The theoretical model was illustrated based on a review of the literature.

To compensate for the missing predictors, the maximum likelihood method (with the missing values) was employed. Stata's sem command is capable of estimating models with missing data using the Full Information Maximum Likelihood estimation (FIML) method ${ }^{16}$ Prior to SEM analysis, multivariate normality was evaluated by examining the normality variables (i.e., estimating Mardia's coefficient of multivariate skewness and kurtosis). To assess the significance of the relationship (the significance level of 5\%), appropriate fitness, Comparative Fit Index (CFI), Tucker-Lewis Index (TLI), Root Mean Square Error of Approximation (RMSEA), and Standardized Root Mean Square Residual (SRMR) were employed. In large sample sizes, $\chi 2 / \mathrm{df}$ is not a suitable choice for model fitness. Hence, for this study, the criteria employed were RMSEA [?] .06 , SRMR [?] .08, and CFI [?] .95. Since latent variables possess several observed variables, it is necessary to assign an arbitrary value, usually 1.0, to the path that links the latent variable to one of its indicator variables to provide a unit of measurement for each latent variable ${ }^{17}$.

Results

The Total Model: This model appeared to fit the data well: CFI (0.969), TLI (0.965), RMSEA (0.051), and SRMR (0.052).

Our findings indicated that in the cohort population $(\mathrm{n}=15006), 32.8 \%$ of participants had at least one chronic disease (table1). The overall MM (i.e., CD [?] 2) was seen in $28.8 \%$ of the XXX cohort population. The most prevalent chronic diseases were obesity (37.6\%), hypertension (20.2\%), depression (17.1\%), and diabetes $(11.6 / \%)$, respectively. In addition, obesity, depression, and diabetes were the most co-occurring CDs in our population (table2).

The baseline characteristics of the participants stratified by the number of chronic diseases are demonstrated 
in table 3. The frequency of chronic diseases was significantly higher in female, urban residents, and unmarried participants. Compared with the subjects in the $3^{\text {rd }}$ tertile METS and the 5 th quintiles of WSI, the prevalence of multimorbidity was significantly higher in subjects with low physical activity $\left(1^{\text {st }}\right.$

tertile METS) and low WSI (poorest; $1^{\text {st }}$ quintiles of WSI).

The SEM diagram with the standardized estimates indicated the total effect of socio-demographic (as predictors) and sleeping habits and physical activity (as the mediator) on the outcome of the study (number of CDs) (Figure 1). In this model, age was inversely associated with sleeping habits $(\mathrm{P}<0.001)$. Moreover, marital status had a positive effect on physical activity $(\mathrm{B}=2.07 ; \mathrm{P}<0.001)$, while the significant negative effect of WSI, age, and gender (female) was observed on physical activity $(\mathrm{P}<0.001)$.

As presented in table 4, age and gender had significant effects on the number of CDs, both directly and through physical activity and sleeping habits $(\mathrm{P}<0.001)$. A significant and direct effect of the number of years of schooling on WSI and the number of CDs were found in our SEM model $(\mathrm{P}<0.001)$. Moreover, it seems that the number of years of schooling indirectly mediated its effect on the number of CDs via physical activity $(\mathrm{B}=-0.11, \mathrm{P}<0.001)$. In specific, people who had higher WSI were more inactive $(\mathrm{B}=-1.09$; $\mathrm{P}<0.001)$.

\section{Discussion}

The findings of this cross-sectional study indicated that $28.8 \%$ of the total population had MM (i.e., [?]2 CDs). A wide range of the prevalence of MM was reported in different studies. The prevalence of MM in the Golestan cohort study, conducted by Malekzadeh et al. in the population aged 40-70 years, was $19.4 \%{ }^{18}$. In another study, according to the dates provided by the health insurance organization, MM has been reported in $21.1 \%$ of the included population ${ }^{19}$. In the aged Kurdish population (i.e., $>50$ years), MM was seen in $36.1 \%$ of participants ${ }^{20}$. Moreover, Aoki et al. studied the pattern of MM in the Japanese population, noting the prevalence of MM to be $29.9 \%$ in all participants, which was higher $(62.8 \%)$ in the older population (i.e., [?] 65 years $)^{21}$. Additionally, in a cohort study in Germany, the prevalence of MM was $67.3 \%$ in the 50-70year-old population ${ }^{22}$. The difference observed in the prevalence of MM in studies may be due to differences in sample size, the types of chronic diseases considered as an MM component, methodology, and ethnicity.

In this study, the most common CDs were obesity, hypertension, depression, and diabetes. Moreover, the most co-occurring CDs were obesity, diabetes, and depression. It should be noted that our findings are in line with previous studies that reported hypertension, diabetes, dyslipidemia, and obesity as the most common CDs in population ${ }^{23}$. Also, the most prevalent CDs reported in the population studied in Blümela et al. were hypertension, arthrosis, diabetes, and depression ${ }^{12}$. In this regard, Read et al. reported that the risk of depression was doubled in patients with MM, compared with subjects without $\mathrm{MM}^{24}$.

As indicated in previous studies, there is a mutual association between depression and CDs ${ }^{25}$. However, the mechanism of this bi-directional relationship is not adequately recognized. Some studies proposed that mechanisms such as the complication of CDs, including disability, decreasing quality of life, ${ }^{26}$ pain, ${ }^{27}$ and belief about the disease and adapting manners are involved in increasing the risk of depression ${ }^{28}$. On the other hand, people with depression are less likely to follow treatment protocols for their CD, which will increase their risk of developing MM and poor control over their illness ${ }^{29}$. Nevertheless, disorders in both metabolic and immune-inflammatory pathways, which occur in many CDs, are associated with depression ${ }^{30}$. Therefore, in patients with CDs, depression should be considered as an important factor.

Studies that evaluated the predictor risk factors of MM using the SEM model are rare. In our SEM model, age and gender influence the number of CDs both directly and indirectly through physical activity and sleeping habits. In studies similar to ours, it has been documented that age and gender were positively associated with $\mathrm{MMs}^{18,19}$ In this regard, Boutayeb et al. reported that females and older people are the predictor factors of MM in WHO Eastern Mediterranean countries ${ }^{31}$. However, in some studies, there are no differences in the prevalence of MM among genders ${ }^{23,32}$ For instance, the EpiChron Cohort Study indicated that the number of CDs increased with age in both genders ${ }^{23}$. 
Our analysis revealed that the number of years of schooling is inversely associated with the number of CDs, which is in line with Blümela et al. that observed an increase of $40 \%$ in the risk of MM among low-educated women who had an unqualified job ${ }^{33}$. Moreover, Johnson-Lawrence et al. showed that the risk of MM in the population of 60-64 years with bachelor's degree (or higher) was lower than less educated people ${ }^{34}$. Since the level of education is a key factor in social/life elements such as employment, health insurance, and housing, lower education levels may lead to lower income, poor living conditions, and psychological stress. These conditions can prevent people from exercising proper health practices such as a healthy diet, physical exercise, and access to preventive health care, resulting in a higher risk of chronic diseases ${ }^{35}$. In this regard, our findings indicated that the number of years of schooling is positively related to WSI. However, it demonstrated a negative effect on physical activity level, confirming the mentioned association between education level, poor social condition, and physical inactivity.

In previous studies, it was reported that socioeconomic status is correlated with MM. In specific, the prevalence of MM was higher in low socioeconomic groups ${ }^{19,36}$ As confirmed in this study, the frequency of MM decreased with increasing WSI, which confirms the findings of the aforementioned studies. Moreover, living in rural areas had a significantly negative effect on WSI, and the number of years of schooling was positively associated with WSI. It has been suggested that the higher number of CDs in the population with low WSI can be attributed to less knowledge about symptoms of CDs, fewer checkups, and the consumption of unhealthy food.

The other finding of the present study indicated that smoking was inversely associated with the number of CDs. We asked the participants about their current smoking status. Therefore, stopping smoking after developing CDs may be one of the reasons for this result ${ }^{37}$. On the other hand, in previous studies, a history of smoking has been documented as a risk factor for $\mathrm{MM}^{18,38}$.

We found that the number of CDs in the active participants ( $3^{\text {rd }}$ tertile METS) and those who sleep 6.6-7.3 hours/day were lower than the inactive population and those who sleep [?]6.5 hours/day the SEM pathway. In other words, physical activity and sleeping habits had a significant effect on the number of CDs. Similar to the present study, Ruiz-Castel et al. noted that the chance of having two, or three or more chronic conditions increased by 7.30 and 6.79 times, respectively, in participants who sleep $<6$ hours/day ${ }^{39}$. Finally, Nicholson et al. reported that the risk of MM was higher in participants who sleep either $<6$ hours/day or $>8$ hours/day ${ }^{33}$.

It appears that the association between sleeping habits and the number of CDs is reciprocal. To elaborate, the sleeping habits in subjects with CDs are subject to change by the pain caused by some CDs, medications/treatments used, and mood disorders ${ }^{40}$. On the other hand, it is well-known that sustained sleep deprivation demonstrates negative effects on cardio-metabolic, endocrine, immune system, and inflammatory pathways ${ }^{41,42}$. Moreover, shorter sleep duration could modify the circadian rhythm and alter the hormonal system (e.g., insulin resistance and decreased leptin $)^{43}$.

The association between physical activity and the number of CDs in the present study is in agreement with Christofoletti et al. In that study, it was reported that the frequency of co-existing CDs was greater among those who had more leisure time and watched TV [?]2 hours/day ${ }^{22}$. Moreover, Ryan et al. noted a significant association between physical activity and $\mathrm{MM}^{44}$.

Physical activity and sleeping habits are mediators, while WSI, the number of years of schooling, marital status, and residence regions (rural) influence the number of CDs through physical activity and sleeping habits.

The main strength of this cohort study is the use of data obtained from a large population. In addition, there are other strengths to this study, including the large number of participants and the data obtained with details of the lifestyle, social, and demographic status of the participants.

Similar to all studies, this study had its limitations, including the type of study (i.e., cross-sectional) and the illnesses expressed by each individual, which is likely to bias the data due to the difference in the level 
of literacy and information retrieval from the participants.

Conclusion

Our study is valuable since it examined the multimorbidity patterns from multiple economic, social, and epidemiologic aspects in parts of XXX where the general population is at an increased risk of depression and components of the metabolic syndrome (obesity, hypertension, and diabetes).

This increased risk is specifically more prevalent among the least disadvantaged population with risk factors such as old age, gender (female), low socioeconomic status, low education levels, improper sleeping habits, inadequate hygiene, and inactivity.

Therefore, adequate sleeping habits and physical activity should be considered effective factors in the health of the population. Since multimorbidity are very common in our population, a comprehensive program should be developed to promote sleep health and reduce multimorbidity.

Another finding in this study indicated depression as the pivotal factor in multidisciplinary patterns, which is closely associated with an increased risk of chronic diseases. Depressed people are at risk of chronic disease. Hence, they should be screened and treated. Finally, it should be noted that the reduction in multimorbidity is possible by promoting public health from an early age and among a wide range of socioeconomic conditions, provided that the necessary support for health is offered to the aging population of XXX.

\section{Declaration}

\section{Funding}

This study was supported by the liver and gastrointestinal diseases research center (Grant number700/108 on 14 March 2016), XXX University of Medical Sciences. The funder had no role on the study design, data analysis, interpreting and writing the manuscript in this study.

\section{Competing interests}

The authors declare that they have no competing interests

\section{Availability of data and materials}

The data that support the findings of this study are available from [Vice Chancellor for Research] but restrictions apply to the availability of these data, which were used under license for the current study, and so are not publicly available. Data are however available from the authors upon reasonable request and with permission of [Vice Chancellor for Research]

\section{Authors' contributions}

-The conception or design of the work: MHS,ARO,EF

-The acquisition, analysis: NG, EF

OR interpretation of data:EF,NG

Drafted the work or substantively revised: EF, AHK, SH, MHS,

All authors have read and approved the manuscript

Reference:

1. Hernandez B, Reilly RB, Kenny RA. Investigation of multimorbidity and prevalent disease combinations in older Irish adults using network analysis and association rules.Scientific Report. 2019;9(1):1-12.

2. Violan C, Foguet-Boreu Q, Flores-Mateo G, et al. Prevalence, determinants and patterns of multimorbidity in primary care: a systematic review of observational studies. PloS One. 2014;9(7):e102149. 
3. Licher S, Heshmatollah A, van der Willik KD, et al. Lifetime risk and multimorbidity of non-communicable diseases and disease-free life expectancy in the general population: a population-based cohort study. PLoS Med. 2019;16(2):e1002741.

4. Timmermans EJ, Hoogendijk EO, Broese van Groenou MI, et al. Trends across 20 years in multiple indicators of functioning among older adults in the Netherlands.Eur J Public Health. 2019;29(6):1096-1102.

5. Jani BD, Hanlon P, Nicholl BI, et al. Relationship between multimorbidity, demographic factors and mortality: findings from the UK Biobank cohort. BMC Med.2019;17(1):74.

6. Volaklis KA, Thorand B, Peters A, et al. Physical activity, muscular strength, and polypharmacy among older multimorbid persons: Results from the KORA-Age study. Scand J Med Scien Sport. 2018;28(2):604612.

7. Villen N, Guisado-Clavero M, Fernandez-Bertolin S, et al. Multimorbidity patterns, polypharmacy and their association with liver and kidney abnormalities in people over 65 years of age: a longitudinal study. BMC Geriatr 2020;20(1):1-13.

8. Yu T, Ter Riet G, Puhan MA, Frei A. Physical activity and risk of comorbidities in patients with chronic obstructive pulmonary disease: a cohort study. NPJ Primary Care Respirat Med. 2017;27(1):1-6.

9. Marques A, Santos DA, Peralta M, Sardinha LB, Valeiro MG. Regular physical activity eliminates the harmful association of television watching with multimorbidity. A cross-sectional study from the European Social Survey. Prev Med.2018;109:28-33.

10. Wang S, Li B, Wu Y, et al. Relationship of sleep duration with sociodemographic characteristics, lifestyle, mental health, and chronic diseases in a large Chinese adult population. J Clin Sleep Med. 2017;13(3):377384.

11. Griffith LE, Gilsing A, Mangin D, et al. Multimorbidity Frameworks Impact Prevalence and Relationships with Patient-Important Outcomes. J Am Geriatr Society.2019;67(8):1632-1640.

12. Blumel JE, Carrillo-Larco RM, Vallejo MS, Chedraui P. Multimorbidity in a cohort of middle-aged women: Risk factors and disease clustering. Maturitas. 2020.

13. Eyowas FA, Schneider M, Yirdaw BA, Getahun FA. Multimorbidity of chronic non-communicable diseases and its models of care in low-and middle-income countries: a scoping review protocol. BMJ Open. 2019;9(10):e033320.

14. Poustchi H, Eghtesad S, Kamangar F, et al. Prospective epidemiological research studies in Iran (the PERSIAN Cohort Study): rationale, objectives, and design. Am J Epidemiol 2018;187(4):647-655.

15. Farhang S, Faramarzi E, Amini Sani N, et al. Cohort Profile: The AZAR cohort, a health-oriented research model in areas of major environmental change in Central Asia.Inter J Epidemiol. 2019;48(2):382$382 \mathrm{~h}$.

16. Medeiros RA. Including Auxiliary Variables in Models with Missing Data Using Full Information Maximum Likelihood. Paper presented at: 2013 Stata Conference2013.

17. Kline RB. Principles And Practice Of Structural Equation Modeling. Guilford publications; 2015.

18. Ahmadi B, Alimohammadian M, Yaseri M, et al. Multimorbidity: epidemiology and risk factors in the Golestan cohort study, Iran: a cross-sectional analysis. Med.2016;95(7).

19. Ebrahimoghli R, Janati A, Sadeghi-Bazargani H, et al. Epidemiology of multimorbidity in Iran: An investigation of a large pharmacy claims database.Pharmacoepidemiol Drug Safety. 2020;29(1):39-47.

20. Aminisani N, Rastgou L, Shamshirgaran SM, Sarbakhsh P, Ghaderi S, Hyde M. Predictors of multimorbidity among the Kurdish population living in the Northwest of Iran. BMC Public Health. 2020;20(1):1-8. 
21. Aoki T, Yamamoto Y, Ikenoue T, Onishi Y, Fukuhara S. Multimorbidity patterns in relation to polypharmacy and dosage frequency: a nationwide, cross-sectional study in a Japanese population. Scientific Report. $2018 ; 8(1): 1-8$.

22. Christofoletti M, Duca GFD, Umpierre D, Malta DC. Chronic noncommunicable diseases multimorbidity and its association with physical activity and television time in a representative Brazilian population. Cadernos De Saude Publica.2019;35:e00016319.

23. Ioakeim-Skoufa I, Poblador-Plou B, Carmona-Pirez J, et al. Multimorbidity Patterns in the General Population: Results from the EpiChron Cohort Study. Inter J Environmental Res Public Health. 2020;17(12):4242.

24. Read JR, Sharpe L, Modini M, Dear BF. Multimorbidity and depression: A systematic review and meta-analysis. J Affective Disorder. 2017;221:36-46.

25. Katon WJ. Epidemiology and treatment of depression in patients with chronic medical illness.Dialogues Clin Neuroscien. 2011;13(1):7.

26. Katon WJ. Clinical and health services relationships between major depression, depressive symptoms, and general medical illness. Biol Psychiatr 2003;54(3):216-226.

27. Bair MJ, Robinson RL, Katon W, Kroenke K. Depression and pain comorbidity: a literature review.Arch Inter Med 2003;163(20):2433-2445.

28. Ziarko M, Mojs E, Piasecki B, Samborski W. The mediating role of dysfunctional coping in the relationship between beliefs about the disease and the level of depression in patients with rheumatoid arthritis. Scientific World J. 2014;2014.

29. Alexopoulos GS, Raue PJ, Sirey JA, Arean PA. Developing an intervention for depressed, chronically medically ill elders: a model from COPD. Inter J GeriatriPsychiat. 2008;23(5):447-453.

30. Penninx BW. Depression and cardiovascular disease: epidemiological evidence on their linking mechanisms. Neuroscien BiobehaviorRev. 2017;74:277-286.

31. Boutayeb A, Boutayeb S, Boutayeb W. Multi-morbidity of non communicable diseases and equity in WHO Eastern Mediterranean countries. Inter J Equity Health.2013;12(1):1-13.

32. Excoffier S, Herzig L, N'Goran AA, Deruaz-Luyet A, Haller DM. Prevalence of multimorbidity in general practice: a cross-sectional study within the Swiss Sentinel Surveillance System (Sentinella). BMJ Open. 2018;8(3):e019616.

33. Nicholson K, Rodrigues R, Anderson KK, Wilk P, Guaiana G, Stranges S. Sleep behaviours and multimorbidity occurrence in middle-aged and older adults: findings from the Canadian Longitudinal Study on Aging (CLSA). Sleep Med.2020;75:156-162.

34. Johnson-Lawrence V, Zajacova A, Sneed R. Education, race/ethnicity, and multimorbidity among adults aged 30-64 in the National Health Interview Survey. SSM - Population Health. 2017;3:366-372.

35. Ross CE, Wu C-l. The links between education and health. Am Sociological Rev. 1995:719-745.

36. Barnett K, Mercer SW, Norbury M, Watt G, Wyke S, Guthrie B. Epidemiology of multimorbidity and implications for health care, research, and medical education: a cross-sectional study. Lancet. 2012;380(9836):37-43.

37. Islami F, Pourshams A, Vedanthan R, et al. Smoking water-pipe, chewing nass and prevalence of heart disease: a cross-sectional analysis of baseline data from the Golestan Cohort Study, Iran. Heart. 2013;99(4):272-278.

38. Chung RY, Mercer S, Lai FT, Yip BH, Wong MC, Wong SY. Socioeconomic determinants of multimorbidity: a population-based household survey of Hong Kong Chinese. PLoS One.2015;10(10):e0140040. 
39. Ruiz-Castell M, Makovski TT, Bocquet V, Stranges S. Sleep duration and multimorbidity in Luxembourg: results from the European Health Examination Survey in Luxembourg, 2013-2015. BMJ Open. 2019;9(8):e026942.

40. He Q, Sun H, Wu X, et al. Sleep duration and risk of stroke: a dose-response meta-analysis of prospective cohort studies. Sleep Med. 2017;32:66-74.

41. Kumari M, Badrick E, Ferrie J, Perski A, Marmot M, Chandola T. Self-Reported Sleep Duration and Sleep Disturbance Are Independently Associated with Cortisol Secretion in the Whitehall II Study. J Clin Endocrinol Metabolism.2009;94(12):4801-4809.

42. Buxton OM, Pavlova M, Reid EW, Wang W, Simonson DC, Adler GK. Sleep restriction for 1 week reduces insulin sensitivity in healthy men. Diabete.2010;59(9):2126-2133.

43. Gangwisch J. Epidemiological evidence for the links between sleep, circadian rhythms and metabolism. Obesity Rev. 2009;10:37-45.

44. Ryan A, Murphy C, Boland F, Galvin R, Smith SM. What Is the Impact of Physical Activity and Physical Function on the Development of Multimorbidity in Older Adults Over Time? A Population-Based Cohort Study. J Gerontol Series A Biol Scien Med Scien. 2018;73(11):1538-1544.

\section{Hosted file}

table99.8.27.docx available at https://authorea.com/users/418210/articles/525034-patternand-predicting-risk-factors-of-multi-morbidity-in-the-azarcohort-population-usingstructural-equation-model

\section{Hosted file}

SEM.docx available at https://authorea.com/users/418210/articles/525034-pattern-andpredicting-risk-factors-of-multi-morbidity-in-the-azarcohort-population-usingstructural-equation-model 